Cite this: Analyst, 2014, 139, 3999

\title{
Electrochemistry provides a point-of-care approach for the marker indicative of Pseudomonas aeruginosa infection of cystic fibrosis patients
}

\begin{abstract}
Jonathan P. Metters, Dimitrios K. Kampouris and Craig E. Banks*
It has recently been demonstrated that 2-aminoacetophenone (2-AA) is a chemical indicator in exhaled air/ breath of Pseudomonas aeruginosa infection associated with progressive life threatening decline of lung function in cystic fibrosis sufferers [Scott-Thomas et al., BMC Pulm. Med., 2010, 10, 56]. Currently the detection of 2-AA involves laboratory based instrumentation such as mass spectrometry and a handheld point-of-care type breath device would be ideal in providing real-time results within seconds to accelerate patient care decision-making processes. To this end, we demonstrate proof-of-concept that the chemical marker 2-AA, indicative of Pseudomonas aeruginosa infection, can be measured using electrochemical based sensing strategies. A range of commercially available electrode substrates are explored demonstrating for the first time that 2-AA is electrochemically active within aqueous based solutions providing an (electro)analytical signal. Glassy carbon, boron-doped diamond and platinum electrodes have been explored towards the electrochemical oxidation of 2-AA. Electrode fouling is observed requiring pre-treatment in the form of mechanical polishing between voltammetric scans and measurements. To alleviate this, screen-printed graphite electrodes are shown to be a more viable option for implementation into breath sensing devices and overcome the fouling problem since due to their low cost and disposable nature, a new electrode can be used for each measurement. The analytical utility of the platinum, screen-printed and boron-doped diamond electrodes were found to correspond to $6.85,7.66$ and $4.86 \mathrm{mM}$ respectively. The challenges associated with the electrochemical sensing of $2-\mathrm{AA}$ in breath that need to be overcome are discussed. This generic approach where electrochemical based technology is used to provide measurements for chemical markers in exhaled air/breath for medical diagnostics termed electrochemical breathprints (ec-breathprints), has the potential to be developed into a hand-held point-of-care breath diagnostic tool for identifying Pseudomonas aeruginosa infection in exhaled air/breath.
\end{abstract}

Received 15th April 2014

Accepted 30th May 2014

DOI: $10.1039 / c 4 a n 00675 \mathrm{e}$

www.rsc.org/analyst

\section{Introduction}

The analysis of exhaled air/breath is becoming widely accepted as a non-invasive and rapid approach for clinical diagnostics in the detection and diagnostics of diseases. ${ }^{1-9}$ So called Breathprints, where unique chemical signatures allow clinical diagnosis to be made, is gaining impetus building upon well-known chemical markers, for example, such as nitric oxide in exhaled breath of asthma sufferers, ${ }^{10,11}$ hydrogen and methane to assess malabsorption of sugars in the small intestine, and the detection of Helicobacter pylori infection involving the ingestion of isotopically-labelled compounds such as carbon-14 or non-

Faculty of Science and Engineering, School of Chemistry and the Environment, Division of Chemistry and Environmental Science, Manchester Metropolitan University, Chester Street, Manchester M1 5GD, Lancs, UK. E-mail: c.banks@mmu.ac.uk; Web: http:// www.craigbanksresearch.com; Fax: +44 (0)1612476831; Tel: +44 (0)1612471196 radioactive carbon- 13 with the detection of the isotope labelled carbon dioxide. ${ }^{12}$ Current research is directed to finding new chemical markers in breath/exhaled air that can provide clinical diagnosis such as in the case of volatile organic compounds for lung cancer, ${ }^{13,14}$ isoprene, acetone, trimethylamine, acetaldehyde, and pentane for non-alcoholic fatty liver disease in children, ${ }^{15}$ isoprene for identifying end-stage renal disease, ${ }^{16}$ acetone for diabetes ${ }^{8}$ and nitric oxide for chronic obstructive pulmonary disease ${ }^{17}$ to name just a few.

It is well established that hydrogen cyanide is a useful biomarker, ${ }^{18-20}$ particularly for the indication of Pseudomonas aeruginosa infection in cystic fibrosis (CF) patients. ${ }^{21}$ The characteristics of $\mathrm{CF}$ are recurrent respiratory infections and chronic inflammation resulting in deterioration of lung function and can eventually end in respiratory failure. ${ }^{21}$ Pseudomonas aeruginosa is reported to be the most important respiratory infection in $\mathrm{CF}$ and can result in a significant increase in patient 
morbidity and mortality. ${ }^{22-24}$ If Pseudomonas aeruginosa infection can be diagnosed early and suitable antibiotic treatment is undertaken, it can become eradicated before the infection becomes chronic. The gold standard diagnosis is the culture of expectorated sputum ${ }^{25}$ but it is reported, especially in young children that patients are unable or unwilling to expectorate sputum and the alternative of cough swabs are unreliable or induced sputum samples which are time consuming, costly and unpleasant. ${ }^{25}$ Consequently mouth and nose exhaled breath is an appealing approach to overcome these clinical sampling problems and provides a simple yet effective non-invasive approach for the identification of Pseudomonas aeruginosa infection in cystic fibrosis patients. ${ }^{21}$

Recently pioneering work from Scott-Thomas and colleagues reported that 2-aminoacetophenone (2-AA) is also a chemical marker in exhaled air/breath for Pseudomonas aeruginosa infection in CF sufferers. ${ }^{26}$ Follow-up work by Gilchrist et al. ${ }^{18}$ using SIFT-MS have independently confirmed that 2-AA can indeed be a useful marker of pulmonary Pseudomonas aeruginosa infection alongside hydrogen cyanide albeit that the concentrations of 2-AA were observed to be lower than previously reported, ${ }^{\mathbf{2 6}}$ though no reason was given.

In this work we demonstrate for the first time that the chemical marker for Pseudomonas aeruginosa infection, ${ }^{26}$ 2-AA, can be electroanalytically detected in aqueous based solutions using electrochemical based technology and due to the electrochemical mechanism, the use of disposable one-shot screenprinted electrochemical sensors are favoured. Such electrodes have the potential to be incorporated into a point-of-care breath device providing useful clinical information of Pseudomonas aeruginosa infection in $\mathrm{CF}$ patients.

\section{Experimental}

All chemicals were of the highest grade available and were used as received (without further purification) from Sigma Aldrich (UK). All solutions were prepared using deionised water of resistivity no less than $18.2 \mathrm{M} \Omega \mathrm{cm}$ and were vigorously degassed prior to electrochemical measurements with high purity, oxygen free nitrogen. For measurements involving carbon dioxide and oxygen the solution was saturated (separately) with the required gas via vigorously bubbling the solution for 20 minutes prior to electrochemical measurement. For the introduction of 2-AA to solutions at fixed concentrations an Owlstone OCG-4 Gas Generator ${ }^{27}$ was utilised.

Voltammetric measurements were carried out using an $\mu$ Autolab III (ECO-Chemie, The Netherlands) potentiostat. All measurements were conducted using either: screen-printed three electrode configuration (see below) with a geometric working electrode area of $3 \mathrm{~mm}$ diameter, boron-doped diamond (BDD), polycrystalline platinum or glassy carbon (GC) electrode. The BDD and GC were mechanically polished/cleaned using a polishing pad and diamond sprays. The platinum electrode was polished using alumina on a soft polishing pad.

Screen-printed carbon-based electrodes (denoted as SPEs) were fabricated in-house with appropriate stencil designs using a microDEK 1760RS screen-printing machine (DEK, Weymouth, UK). Note that this screen-printed electrode design has been previously reported ${ }^{28-32}$ "as is" without electrode pre-treatment or modification in various electroanalytical endeavours. For fabrication of the SPEs, first a carbon ink formulation (Product Code: C2000802P2; Gwent Electronic Materials Ltd, UK) utilised for the efficient connection of all three electrodes and the electrode material for both the working and counter electrodes was screen-printed onto a polyester (Autostat, 250 micron thickness) flexible film. The carbon ink layer was cured in a fan oven at 60 degrees for 30 minutes. Next a silver/silver chloride reference electrode was included by screen-printing $\mathrm{Ag} / \mathrm{AgCl}$ paste (Product Code: C2040308P2; Gwent Electronic Materials Ltd, UK) onto the polyester substrates which was subsequently cured once more in a fan oven at 60 degrees for 30 minutes. Finally, a dielectric paste (Product Code: D2070423P5; Gwent Electronic Materials Ltd, UK) was then printed onto the polyester substrate to cover the connections and define the active electrode areas including that of the working electrode $(3 \mathrm{~mm}$ diameter). After curing at 60 degrees for 30 minutes the SPEs are ready to be used. These electrodes have been characterised electrochemically in a prior paper and have heterogeneous rate constants of $1.08 \times 10^{-3} \mathrm{~cm} \mathrm{~s}^{-1} .^{33}$ The reproducibility and repeatability of the fabricated batches of electrodes were explored through comparison of cyclic voltammetric responses using $1 \mathrm{mM}$ hexaammine-ruthenium(III) chloride/0.1 M KCl. Analysis of the voltammetric data revealed the \% relative standard deviation to correspond to no greater than $0.82 \%(N=20)$ and $0.76 \%(N=3)$ for the reproducibility and repeatability of the fabricated SPEs for use in electroanalysis.

\section{Results \& discussion}

We first explore the validity of the electrochemical determination of $10 \mathrm{mM}$ 2-AA using glassy carbon (GC) and boron-doped diamond (BDD) electrodes in a $\mathrm{pH} 7$ phosphate buffer solution. The GC electrode was found to provide an electrochemical signature with intriguing voltammetry noted. Oxidative voltammetric peaks were observed at potentials of $\sim+0.07 \mathrm{~V}$, $+0.26 \mathrm{~V}$ and $+0.39 \mathrm{~V}(v s$. $\mathrm{Ag} / \mathrm{AgCl})$ while a single reduction peak was observed at $\sim-0.11 \mathrm{~V}(v s$. $\mathrm{Ag} / \mathrm{AgCl})$. It was observed that upon potential cycling each of the voltammetric peaks were noted to grow. Since the peaks grow in magnitude rather than reduce, such observations suggest that a conducting polymer is being formed upon the electrode surface. To the best of our knowledge there is only one electrochemical report on the electrochemistry of 2-AA and related compounds which reports that 2-AA can readily polymerise upon a GC electrode surface; ${ }^{34}$ such work verifies our observations. In addition to GC being explored as an electrode material, a BDD electrode was also explored; such an electrode is routinely used in the field of electrochemistry since its robust surface has been reported to exhibit less surface fouling compared to other carbon based electrode substrates. The response of the BDD electrode towards the electrochemical detection of 2-AA is shown in Fig. 1 which reveals that there are three oxidation peaks observed at $+0.17,+0.34$ and $+0.39 \mathrm{~V}(v s$. $\mathrm{Ag} / \mathrm{AgCl})$ and a single reduction. 


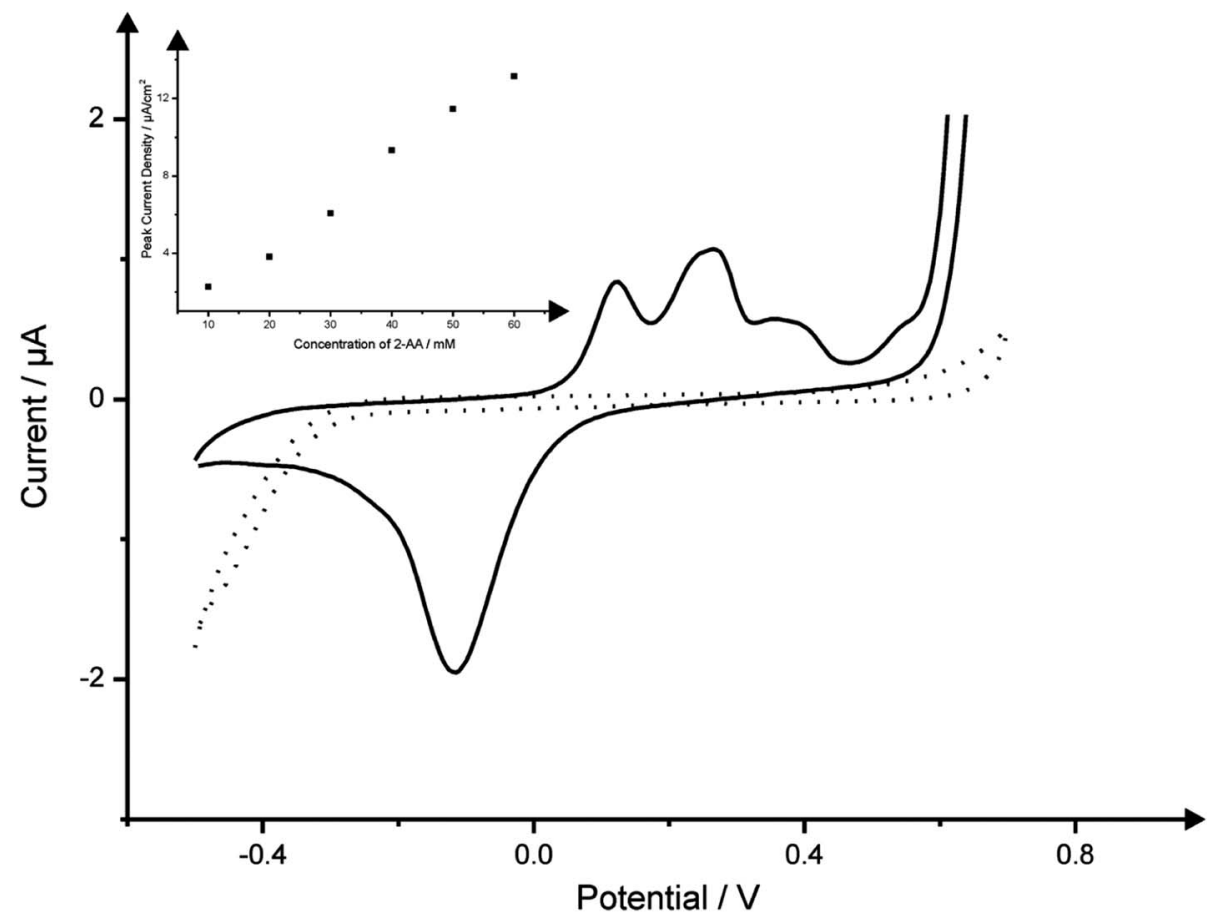

Fig. 1 Cyclic voltammetric measurements recorded in the absence (dotted line) and presence (solid line) of $60 \mathrm{mM} 2-\mathrm{AA}$ in a pH 7 phosphate buffer solution using a boron-doped diamond electrode. Scan rate: $100 \mathrm{mV} \mathrm{s}^{-1}$ (vs. Ag/AgCl). Inset: a typical calibration plot corresponding to additions of 2-AA over the range of 10 to $60 \mathrm{mM}$ using peak $+0.34 \mathrm{~V}$. Note that the electrode was polished between each measurement.

Again, potential cycling revealed that the magnitude of each of the described voltammetric peaks increased but the magnitude of which was less than that observed at the GC electrode confirming less "fouling", but the response is still far from ideal.

In order for the BDD electrode to be useful in electroanalysis, mechanical polishing between voltammetric scans and additionally between measurements of 2-AA concentrations is required. The electroanalytical response of the $\mathrm{BDD}$ was explored with additions of 2-AA made into a pH 7 PBS using the BDD electrode which was mechanically polished between each addition and voltammetric measurement. Using the voltammetric peak observed at $\sim 0.34 \mathrm{~V}$ ( $v s$. $\mathrm{Ag} / \mathrm{AgCl})$, analysis of the voltammetric peak height $\left(I_{\mathrm{P}}\right)$ as a function of concentration is shown in the insert of Fig. 1 which reveals a linear range from 10 to $60 \mathrm{mM}\left(I_{\mathrm{P}}(\mathrm{A})=1.69 \times 10^{-8}\left(\mathrm{~A} \mathrm{mM}^{-1}\right)+5.33 \times 10^{-8}(\mathrm{~A}) ; R^{2}=\right.$ $0.98 ; N=6$ ) beyond which the response is observed to plateau.

Since the voltammetric response at carbon electrodes appear to involve the formation of a conducting polymer, the study was expanded towards the potential utilisation of a noble metal electrode substrate, namely polycrystalline platinum. As depicted in Fig. 2 the successful electrochemical determination of 2AA is possible using the platinum electrode with the oxidation potential noted to occur at $\sim+0.88 \mathrm{~V}$ ( $v s$. $\mathrm{Ag} / \mathrm{AgCl})$. While only one peak was initially observed, additional voltammetric peaks appear in the anodic region upon potential cycling with a reduction in the magnitude of the main peak; thus it is necessary to mechanically polish the electrode between measurements. The electroanalytical response at the platinum electrode was explored with the voltammetric response monitored as a function of 2-AA concentration additions into a pH 7 PBS where, as shown in the insert of Fig. 2, a linear range of voltammetric peak height against concentration is observed from 10 to 60 $\mathrm{mM}\left(I_{\mathrm{P}}(\mathrm{A})=1.35 \times 10^{-6}\left(\mathrm{~A} \mathrm{mM}^{-1}\right)+2.46 \times 10^{-5}(\mathrm{~A}) ; R^{2}=0.99\right.$; $N=6)$.

Next attention was turned to exploring the response of SPEs; such electrodes can be used as one-shot electrochemical sensors, that is, there is no need for electrochemical pre-treatment in the form of mechanical polishing between measurements greatly simplifying the analytical protocol. ${ }^{28-32}$ Additionally SPEs are more suited to point-of-care sensors than other solid electrode substrates such as GC, BDD and platinum due to the significant cost benefits of the SPEs. Fig. 3 displays typical optical and SEM images of the SPEs used in this work where a well-defined electrode surface is observed, consisting of randomly orientated graphite particles bound together with an inert polymeric binder which is free from cracks and holes. Note the complete sensor depicted in Fig. 3 and utilised herein comprises the dimensions of $4 \mathrm{~cm} \times 0.8 \mathrm{~cm}$. Fig. 4 shows the voltammetric response of 2-AA using a SPE. Inspection of Fig. 4 reveals that the electrochemical oxidation of 2-AA occurs using the SPEs at a potential of $\sim+0.42 \mathrm{~V}(v s . \mathrm{Ag} / \mathrm{AgCl})$ with the peak for the reduction of the oxidised product occurring at a potential of $\sim-0.51 \mathrm{~V}$ ( $v s$. $\mathrm{Ag} / \mathrm{AgCl})$. Additionally there is a small oxidation wave at $\sim-0.26 \mathrm{~V}$ ( $v s$. $\mathrm{Ag} / \mathrm{AgCl}$ ). Upon consecutive potential cycling, using the same electrode, it was noted that each of the voltammetric peaks increased in magnitude initially before plateauing off after $\sim 4$ scans. However such fouling can be avoided through the use of a new electrode for each 


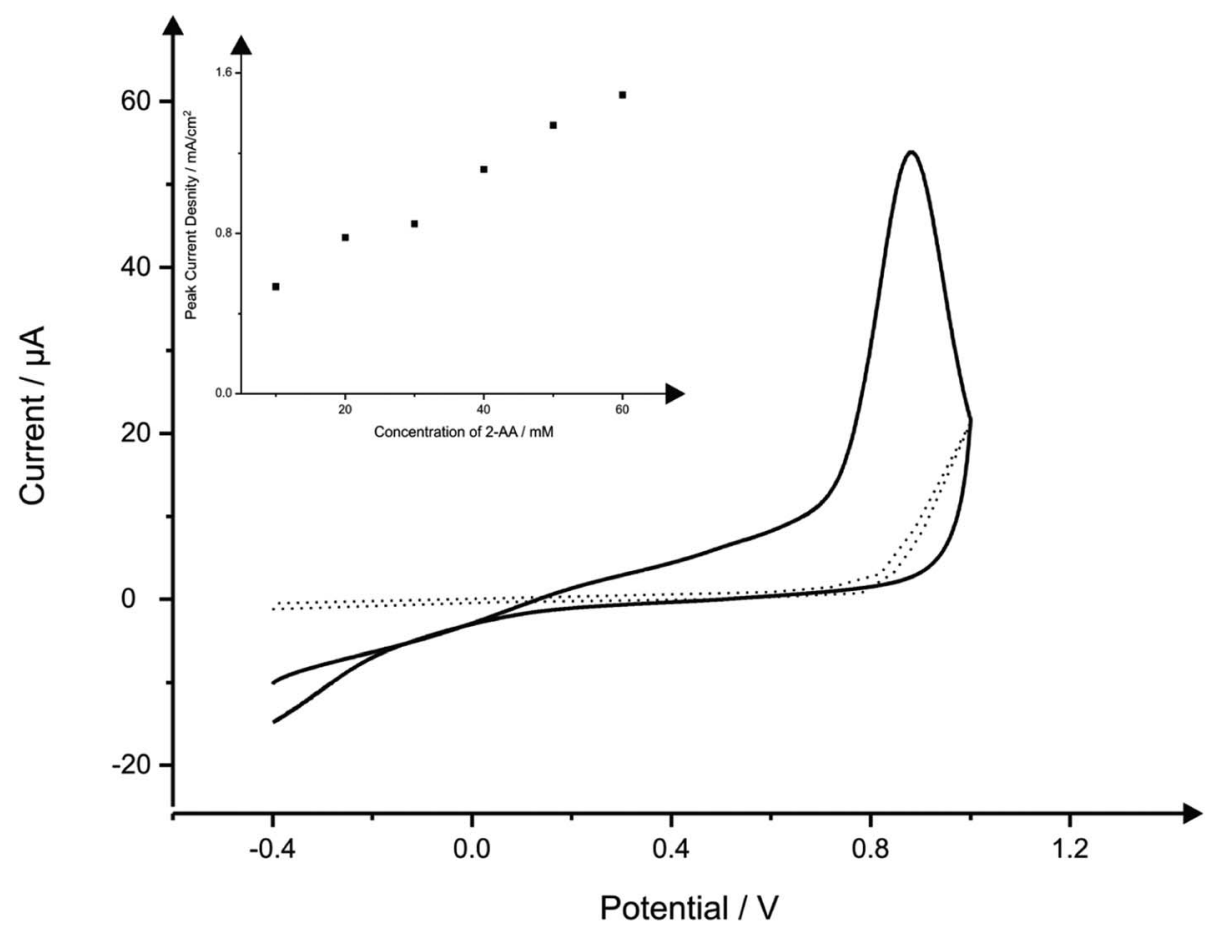

Fig. 2 Cyclic voltammetric measurements recorded in $10 \mathrm{mM} \mathrm{2-AA}$ in a pH 7 phosphate buffer solution using a polycrystalline platinum electrode (solid line). Scan rate: $100 \mathrm{mV} \mathrm{s}^{-1}$ (vs. Ag/AgCl). Inset: typical calibration plots corresponding to additions of 2-AA over the range of 10 to $60 \mathrm{mM}$ using a polycrystalline platinum macroelectrode. Note that the electrode was polished between each measurement.

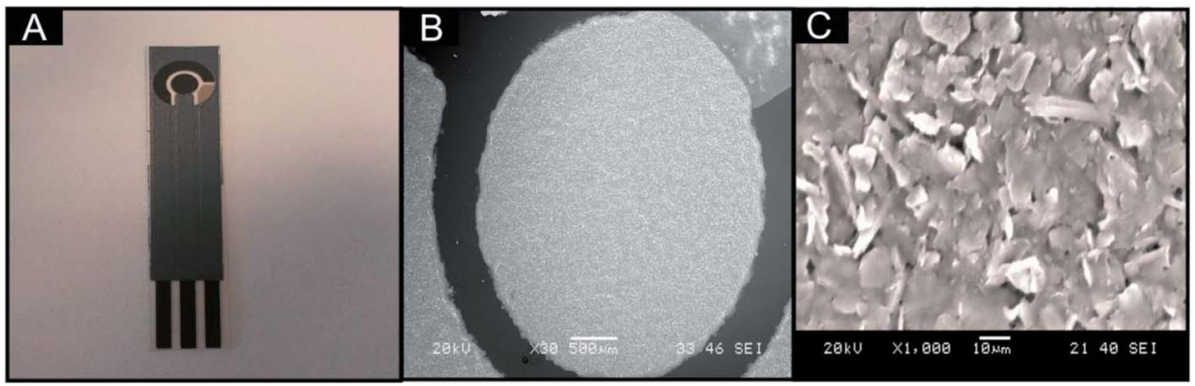

Fig. 3 An optical image (A) and SEM images at increasing magnifications of a graphite screen-printed sensor (SPE).

measurement which is possible due to the low-cost nature of the screen-printed electrodes. Next scan rate studies were performed using the SPEs where analysis of the voltammetric peak height was performed in terms of the peak current and peak potential. A plot of peak current $\left(I_{\mathrm{P}}\right)$ of the peak observed at $\sim+0.42 \mathrm{~V}$ (vs. $\mathrm{Ag} / \mathrm{AgCl})$ against square root of the applied scan rate was found to be linear over the range $5-200 \mathrm{mV} \mathrm{s}^{-1}$ $\left(I_{\mathrm{P}}(\mu \mathrm{A})=5.92 \times 10^{-6}\left(\mathrm{~A} /\left(\mathrm{V} \mathrm{s}^{-1}\right)^{1 / 2}\right)-6.5 \times 10^{-6}(\mathrm{~A}) ; R^{2}=0.98 ;\right.$ $N=7)$ suggesting a diffusional rather than a surface controlled process, while the peak potential was found to shift to high potentials with scan rate indicating an irreversible electrochemically process.

Having determined that the analyte, 2-AA, was electrochemically active at the SPEs, further information into the electrochemical mechanism was sought. Cyclic voltammetric measurements were conducted using the SPE sensor at a fixed concentration of 2-AA using solutions of differing pH's over the range 2.3 to 10 . A plot of $\mathrm{pH}$ versus voltammetric peak potential (oxidation) yielded a line of best-fit equation of $E_{\mathrm{P}}(\mathrm{V})=-0.028$ $(\mathrm{V}$ per $\mathrm{pH})+0.60(\mathrm{~V}) ; R^{2}=0.99$. Note, based on this experimentally deduced gradient of $28.2 \mathrm{mV}$ per decade, such a value is close to that expected for 1 proton and 2 electron process (30 $\mathrm{mV}$ per $\mathrm{pH}$ unit at $25^{\circ} \mathrm{C}$ ). While the exact mechanism is unknown at this stage it likely involves the electrochemical oxidation of the amino group which forms a polymer based on a head-to-tail mechanism; such work has been reported before on glassy carbon electrodes ${ }^{34}$ and also explains why polishing is needed in-between voltammetric measurements; one-shot economical SPEs alleviate this problem.

The electroanalytical response of the SPEs towards 2-AA was last explored. Additions of 2-AA were made into a solution of $\mathrm{pH}$ 7.4 PBS where, as depicted in Fig. 4, (inset) linearity was 


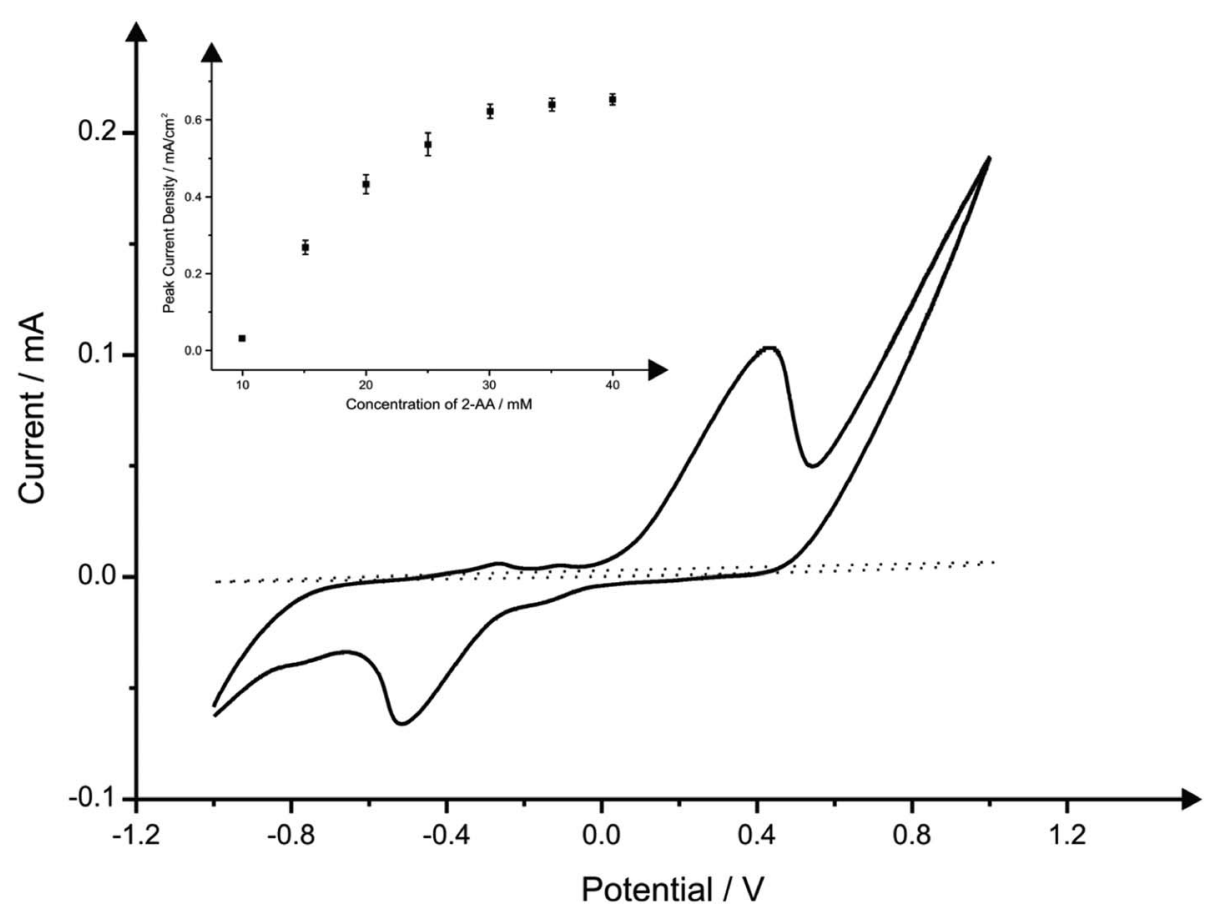

Fig. 4 Cyclic voltammetric measurements recorded in the absence (dotted line) and presence (solid line) of $10 \mathrm{mM} 2-\mathrm{AA}$ in a pH 7 phosphate buffer solution using a graphite screen-printed electrode. Scan rate: $100 \mathrm{mV} \mathrm{s}^{-1}$ (vs. Ag/AgCl). Inset: a typical calibration plot corresponding to additions of 2-AA over the range of 10 to $40 \mathrm{mM}$. Note that each data point is a new sensor each time.

observed $\left(I_{\mathrm{P}}(\mathrm{A})=1.41 \times 10^{-6}\left(\mathrm{~A} \mathrm{mM}^{-1}\right)+2.98 \times 10^{-6}(\mathrm{~A}) ; R^{2}=\right.$ $0.87 ; N=7$ ) over a concentration range of 10 to $30 \mathrm{mM} 2$-AA where upon further additions the response is seen to plateau, likely due to saturation of the analyte upon the electrode surface or arises from other factors; it is important to note that each point on the insert of Fig. 4 is a new electrode and alleviates the need for mechanical polishing between measurements as observed for the other electrode substrates explored in this study.

In comparison of the three materials explored the polycrystalline platinum macroelectrode offered greatest sensitivity, followed by the SPEs, while the BDD was noted to offer voltammetric peaks of diminished stature. Additionally the limits of detection $(3 \sigma)$ for the determination of 2-AA at the polycrystalline platinum macroelectrode, SPEs and BDD electrode were calculated to be $6.85,7.66$ and $4.86 \mathrm{mM}$ respectively. We note however that for potential point-of-care measurements, the SPEs are more ideally suited, especially given their low cost due to scales of economy.

When considering the potential expansion of the electrochemical protocol towards the determination of 2-AA in breath samples, exploration of the potential interferences, namely carbon dioxide $\left(\mathrm{CO}_{2}\right)$ and oxygen $\left(\mathrm{O}_{2}\right)$ were conducted since they are known to be electrochemically active. Separate electrochemical measurements (not shown) were carried using out an SPE in a solution of pH 7.4 PBS containing $10 \mathrm{mM}$ 2-AA with the two gases being bubbled individually into the solutions (to saturation) whereby it was noted that no change in the voltammetric peak potential nor peak magnitude was noted. In order to try and replicate breath samples, 2AA was explored as a vapour. A 2-AA gas permeation tube ${ }^{27}$ was constructed with 2-AA and used with a commercially available gas generator (see Experimental) presented in the vapour phase into a $1 \mathrm{~mL} \mathrm{pH} 7.4$ PBS with bubbling undertaken for 20 seconds and 80 seconds. The voltammetric signature was explored which was found to approximately quadruple following the longer bubbling duration. It is noted that while a voltammetric response is possible from gaseous 2-AA, extensive studies measuring 2-AA in real breath will need to be undertaken and represents a significant challenge; see the Conclusions section for more details on endeavor.

\section{Conclusions}

In summary we have demonstrated that the chemical marker indicative of Pseudomonas aeruginosa infection, $2-\mathrm{AA},{ }^{26}$ can be electrochemically detected at a range of electrode substrates and can provide (electro)analytically useful measurements in aqueous based solutions. Of particular note is that SPEs can be used which alleviate the need for mechanical polishing between measurements. For the application into a point-of-care device, SPEs are ideally suited due to their relatively low cost and disposable one-shot nature.

The challenges faced in translating an electrochemical sensing approach to that of measuring markers in exhaled air/ breath are significant. Firstly, more clinical trials on a large population are clearly needed to confirm the exact levels or expected distribution of 2-AA which are medically relevant due to recent discrepancies between research groups; ${ }^{6,18}$ recent results report that the quantity of 2-AA produced varies 
according to the Pseudomonas aeruginosa genotype and the interaction of genotype and phenotype. ${ }^{18}$ Secondly, since it is known that bacteria produce a suit of relatively similar compounds in complex mixtures ${ }^{35}$ more extensive clinical trials will reveal if there are other potential electrochemically active interferents. Thirdly, the role of food sources, beverages, cosmetics or medications which can give rise to false positive have been explored but further extensive work is likely required. ${ }^{36}$ Thirdly, can nasal breath also be used as is the case for other well documented markers. ${ }^{37}$ However the main challenge faced to make this into a truly point-of-care breath device involves developing breath sample collection and presentation to the electrode surface/solution. In doing this, 2-AA in the gaseous breath needs to be presented into an aqueous solution for the electrochemical measurement to be conducted and this will dilute the concentration of the marker (which will vary depending on the chosen solution volume) and the choice of solvent (aqueous or even non-aqueous) will determine the solubility of 2-AA with a solvent that has a high solubility favoured. At present the concentrations accessible using the electrochemical approach are significantly different (higher) from that reported for 2-AA in exhaled air/breath and in the headspace of Pseudomonas aeruginosa cultures; ${ }^{18}$ in the latter an average of 1.8 (range $0.1-13.8$ ) ppbv has been reported. Consequently a pre-concentration approach will be required with multiple breath samples likely needed; as such this might be unacceptable in a clinical diagnostic test; all such challenges are currently being addressed.

\section{References}

1 B. Buszewski, M. Kesy and T. Ligor, Biomed. Chromatogr., 2007, 21, 553-566.

2 H. Haick, Y. Y. Broza, P. Mochalski, V. Tuzsanyi and A. Amann, Chem. Soc. Rev., 2014, 43, 1423-1449.

3 S. A. Kharitonov and P. J. Barnes, Am. J. Respir. Crit. Care Med., 2001, 163, 1693-1722.

4 S. A. Kharitonov and P. J. Barnes, Biomarkers, 2002, 7, 1-32. 5 A. Manolis, Clin. Chem., 1983, 29, 5-15.

6 W. Miekisch, J. K. Schubert and G. F. Noeldge-Schomburg, Clin. Chim. Acta, 2004, 347, 25-39.

7 D. Smith and P. Spanel, Mass Spectrom. Rev., 2005, 24, 661700.

8 Z. Wang and C. Wang, J. Breath Res., 2013, 7, 037109.

9 J. Huang, S. Kumar, N. Abbassi-Ghadi, P. Spanel, D. Smith and G. B. Hanna, Anal. Chem., 2013, 85, 3409-3416.

10 M. Elliot, S. L. Heltshe and D. C. Stamey, Clin. Exp. Allergy, 2013, 43, 1351.

11 M. R. McCurdy, A. Sharafkhaneh, H. Abdel-Monem, J. Rojo and F. K. Tittel, J. Breath Res., 2011, 5, 016003.

12 F. Megraud, Gut, 2004, 53, 1374-1384.

13 P. Mazzone, X. Wang, P. Rhodes, R. Martino, S. Lim, M. Beukeman, M. Seeley, H. Choi and J. Jett, Chest, 2013, 144, 645A.

14 M. Phillips, N. Altorki, J. H. Austin, R. B. Cameron, R. N. Cataneo, J. Greenberg, R. Kloss, R. A. Maxfield,
M. I. Munawar, H. I. Pass, A. Rashid, W. N. Rom and P. Schmitt, Cancer Biomarkers, 2007, 3, 95-109.

15 N. Alkhouri, F. Cikach, K. Eng, J. Moses, N. Patel, C. Yan, I. Hanouneh, D. Grove, R. Lopez and R. Dweik, Eur. J. Gastroenterol. Hepatol., 2014, 26, 82-87.

16 P. Lirk, F. Fodrogi, H. R. Greiner, H. Ulmer and J. Rieder, Nephrol., Dial., Transplant., 2003, 18, 937-941.

17 A. F. Gelb, P. J. Barnes, S. C. George, F. L. Riccardolo, G. DiMaria and N. Zamel, J. Breath Res., 2012, 6, 047101.

18 F. J. Gilchrist, H. Sims, A. Alcock, J. Belcher, A. M. Jones, D. Smith, P. Spanel, A. K. Webb and W. Lenney, Anal. Methods, 2012, 4, 3661-3665.

19 F. J. Gilchrist, H. Sims, A. Alock, A. M. Jones, R. J. BrightThomas, D. Smith, P. Spanel, A. K. Webb and W. Lenney, J. Clin. Microbiol., 2013, 51, 3849-3851.

20 P. Spanel, T. Wang and D. Smith, Rapid Commun. Mass Spectrom., 2004, 18, 1869-1873.

21 D. Smith, P. Spanel, F. J. Gilchrist and W. Lenney, J. BreathRes., 2013, 7, 044001.

22 C. Aebi, R. Bracher, S. Liechti-Gallati, H. Tschappeler, A. Rudeberg and R. Kraemer, Eur. J. Pediatr., 1995, 154, S69-S73.

23 T. A. Douglas, S. Brennan, S. Gard, L. Berry, C. Gangell, S. M. Stick, B. S. Clements and P. D. Sly, Eur. Respir. J., 2009, 33, 305-311.

24 N. H. Valerius, C. Koch and N. Hoiby, Lancet, 1991, 338, 725. 25 M. Junger, W. Vautz, M. Kuhns, L. Hofmann, S. Ulbricht, J. I. Baumbach, M. Quintel and T. Perl, Appl. Microbiol. Biotechnol., 2012, 93, 2603.

26 A. J. Scott-Thomas, M. Syhre, P. K. Pattemore, M. Epton, R. Laing, J. Pearson and S. T. Chambers, BMC Pulm. Med., 2010, 10, 56.

27 Owlstone, accessed April 2014, http:/www.owlstonenanotech. com/permeation-tubes/make-your-own-permeation-tubes.

28 N. A. Choudry, D. K. Kampouris, R. O. Kadara and C. E. Banks, Electrochem. Commun., 2010, 12, 6-9.

29 P. M. Hallam, D. K. Kampouris, R. O. Kadara and C. E. Banks, Analyst, 2010, 135, 1947.

30 M. Khairy, D. K. Kampouris, R. O. Kadara and C. E. Banks, Electroanalysis, 2010, 22, 2496.

31 A. V. Kolliopoulos, J. P. Metters and C. E. Banks, Anal. Methods, 2013, 5, 3490.

32 A. V. Kolliopoulos, J. P. Metters and C. E. Banks, Anal. Methods, 2013, 5, 851-856.

33 J. P. Smith, J. P. Metters, D. K. Kampouris, C. LledoFernandez, O. B. Sutcliffe and C. E. Banks, Analyst, 2013, 138, 6185-6191.

34 N. V. Stratan, E. P. Kovlchuk and J. Blazjowski, Russ. J. Gen. Chem., 2004, 74, 1900.

35 L. D. J. Bos, P. J. Sterk and M. J. Schultz, PLoS Pathog., 2013, 9, e1003311.

36 A. Scott-Thomas, J. Pearson and S. Chambers, J. Breath Res., 2011, 5, 046002.

37 A. Amann and D. Smith, Volatile Biomarkers: Non-Invasive Diagnosis in Physiology and Medicine, Elsevier, 2013. 